\title{
Experimental Demonstration of Capacity-Achieving Phase-Shifted Superposition Modulation
}

Estaran Tolosa, Jose Manuel; Zibar, Darko; Caballero Jambrina, Antonio; Peucheret, Christophe; Tafur Monroy, Idelfonso

Published in:

39th European Conference and Exhibition on Optical Communication (ECOC 2013)

Link to article, DOI:

10.1049/cp.2013.1485

Publication date:

2013

Link back to DTU Orbit

Citation (APA):

Estaran Tolosa, J. M., Zibar, D., Caballero Jambrina, A., Peucheret, C., \& Tafur Monroy, I. (2013). Experimental Demonstration of Capacity-Achieving Phase-Shifted Superposition Modulation. In 39th European Conference and Exhibition on Optical Communication (ECOC 2013) (pp. We.4.D.5). IEEE.

https://doi.org/10.1049/cp.2013.1485

\section{General rights}

Copyright and moral rights for the publications made accessible in the public portal are retained by the authors and/or other copyright owners and it is a condition of accessing publications that users recognise and abide by the legal requirements associated with these rights.

- Users may download and print one copy of any publication from the public portal for the purpose of private study or research.

- You may not further distribute the material or use it for any profit-making activity or commercial gain

- You may freely distribute the URL identifying the publication in the public portal 


\title{
Experimental Demonstration of Capacity-Achieving Phase-Shifted Superposition Modulation
}

\author{
Jose Estaran, Darko Zibar, Antonio Caballero, Christophe Peucheret, Idelfonso Tafur Monroy \\ DTU Fotonik, Technical University of Denmark, DK-2800 Kgs. Lyngby, jome@fotonik.dtu.dk
}

\begin{abstract}
We report on the first experimental demonstration of phase-shifted superposition modulation (PSM) for optical links. Successful demodulation and decoding is obtained after $240 \mathrm{~km}$ transmission for 16-, 32- and 64-PSM.
\end{abstract}

\section{Introduction}

In the quest for increasing the capacity of optical fiber transmission links, high-order modulation formats are receiving enormous attention as spectrally efficient solutions ${ }^{1}$. Even though great effort has been devoted to the development and demonstration of very highly efficient formats, the uniform distribution of the symbols on their constellation diagrams is sub-optimum with respect to Shannon's theorem. Several modulation schemes have arisen ${ }^{2,3}$ that cleverly combine coding and high-order mapping formats in order to shape the constellations and provide graceful all-in-one solution to obtain spectrally and power efficient transmission and effectively approach the ultimate channel capacity limit. Recently, iterative polar modulation (IPM) has been experimentally demonstrated for coherent OFDM based optical communication systems, giving up to $1.2 \mathrm{~dB}$ shaping gain and 11.15$\mathrm{b} / \mathrm{s} / \mathrm{Hz}$ intrachannel spectral efficiency ${ }^{4}$.

In this context, superposition coded modulation (SCM) with phase-shifted mapping $(\mathrm{PSM})^{5}$ has been proposed as a promising highrate transmission model to approach the ultimate channel capacity while offering enhanced simplicity and flexibility at the transmitter and receiver sides.

In this paper, the first experimental demonstration of 16-, 32- and 64-level SCMPSM generation, demodulation and decoding for single carrier polarization multiplexed optical communication system is reported. The system performance is investigated in terms of signalto-noise ratio. Finally, a transmission distance of $240 \mathrm{~km}$ is achieved for up to 64-level PSM.

\section{Superposition coded modulation (SCM) with phase-shifted mapping (PSM)}

Rather than performing a nonlinear bit-to-symbol mapping, the symbols are designed in SCM by linearly superimposing a certain number of independent, coded and complex-weighted binary streams ${ }^{5}$. The power and phase of the complex weights are determined by the mapping scheme. In the case of PSM, constant power and uniformly distributed phases across the branches are used.

Despite the central limit theorem not being applicable due to the different phase shifts between the branches, the in-phase and quadrature components are statistically independent. This fact allows the passive and simple generation of geometrically quasiGaussian constellations (see Fig. 1) that arbitrarily approach the ultimate shaping gain for a sufficiently large number of layers and particular values of signal-to-noise ratios (SNRs) (see Fig 2). These are called capacity-achieving modulation formats.

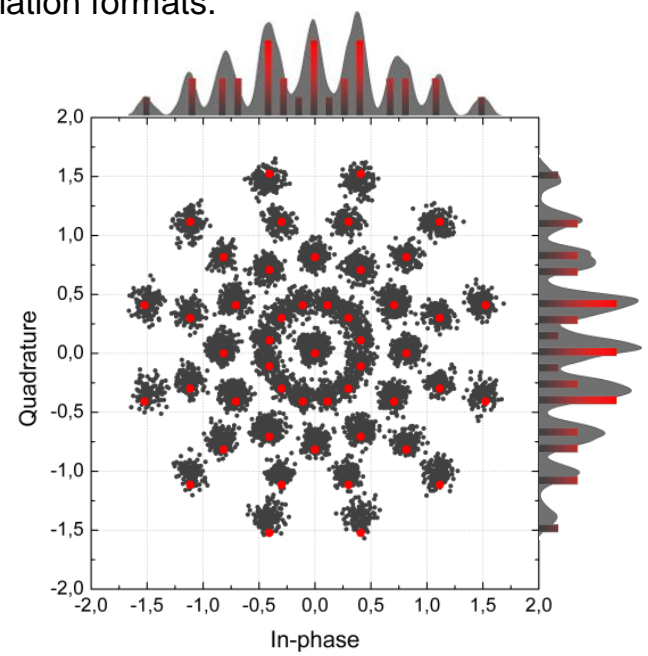

Fig. 1: 64-PSM signal constellation and the in-phase histogram. Ideal (red) and recovered optical back-toback (gray).

Fig. 1 shows an example of recovered optical back-to-back constellation employing 64-PSM together with the ideal constellation points. On the borders, the in-phase and quadrature Gaussian-like histograms of the measured data and ideal data are shown.

The interlayer interference caused by the superposition operation, often gives rise to nonbijective correspondences between symbols and bit tuples. This virtual reduction of cardinality results to be beneficial for some particular mappings where the Euclidean distance among certain adjoining symbols is increased, decreasing then the overall receiver sensitivity. 


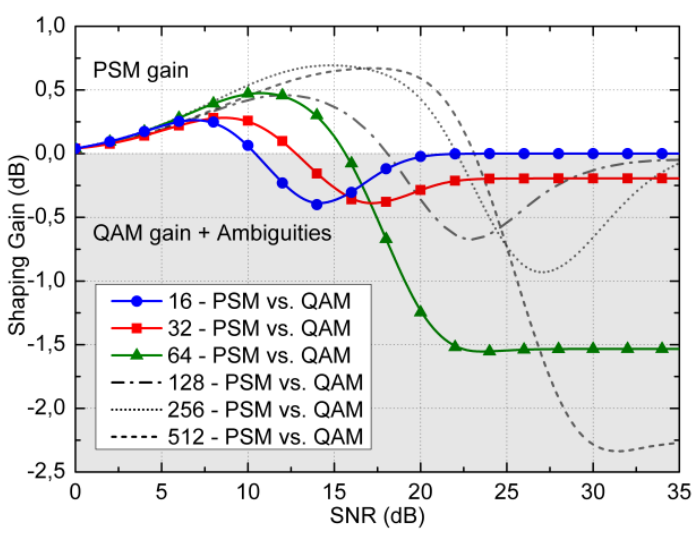

Fig. 2: Shaping gain of PSM vs. QAM for different modulation levels.

Fig. 2 presents the shaping gains for diverse PSM orders over QAM. If the generated constellation is bijective (e.g. 16-PSM), QAM outperforms PSM in a range of SNR values before the shaping gain stabilizes at $0 \mathrm{~dB}$ where maximum transmission capacities are expected to be reached (highest SNRs). In the case of ambiguous mapping (e.g. 32- and 64-PSM) the gain behavior differs at high SNRs. The reduced cardinality of these constellations will prevent the system form attaining the maximum transmission capacity. Such ambiguities are resolved in the receiver side by forcing the demapper and decoder to share extrinsic information repeatedly in an iterative structure that becomes fundamental. This is dubbed "turbo processing"4. Seizing upon the characteristic fact that PSM can be modeled as a Markov process, the Bahl-Cocke-JelinekRaviv (BCJR) algorithm provides the necessary bit-wise information for the demapper to separate the layers at a lower computational cost as compared to the general a posteriori probability (APP) algorithm required when other modulation formats are used.

\section{Experimental Setup}

The schematic of the experimental setup is shown in Fig. 3. At the transmitter, a $1550 \mathrm{~nm}$ external-cavity laser (ECL) with $\sim 100 \mathrm{kHz}$ linewidth is used as the light source. The output of the laser is modulated by an optical push-pull I/Q modulator, which is directly driven from the outputs $\left(2 \mathrm{~V}_{\mathrm{pp}}\right)$ of a 10-bit resolution arbitrary waveform generator (AWG) employed for electrical data signal generation. The sampling rate and the baudrate are fixed over the entire experiment at $12 \mathrm{GS} / \mathrm{s}$ and $6 \mathrm{GBaud}(2 \mathrm{~S} / \mathrm{sym})$, respectively. Pseudo-random binary sequences (PRBS) of length $2^{15}-1$ were digitally processed to generate the waveforms. The processing module consists of: encoding, interleaving, upsampling and pulse shaping. Firstly, 150000 bits are encoded block-wise in a single-level strategy with a $1 / 2$ rate convolutional code and $(5,7)$ polynomial generator. Afterwards, a half-random interleaving is performed (the block lengths vary within 10000-15000 bits depending on the modulation level). The coded sequence is rendered parallel, offering further scrambling and preparing the data for the superposition coded mapping (see next section). After the $\mathrm{I} / \mathrm{Q}$ modulator, polarization division multiplexing (PDM) is emulated. The transmitter output is amplified then launched into an uncompensated fiber link for transmission. The link is made of 3 spans of $80 \mathrm{~km}$ standard singlemode fiber (SSMF). Erbium-dopped fiber amplifiers (EDFAs) are used after each span.

At the receiver side, the incoming modulated optical data signal is coherently mixed with the local oscillator (LO, ECL with $\sim 100 \mathrm{kHz}$ linewidth). Afterwards, the four outputs from the four balanced photodiodes are sampled at 40 $\mathrm{GS} / \mathrm{s}$ and acquired by digital storage oscilloscope (DSO) with $13 \mathrm{GHz}$ bandwidth for offline processing. The digital signal processing (DSP) comprises the following modules: I/Q imbalance compensation, chromatic dispersion (CD) compensation, clock recovery, polarization de-multiplexing, carrier recovery, expectation maximization (EM) and iterative receiver. In order to perform polarization de-multiplexing the constant modulus algorithm (CMA) is firstly used for pre-convergence followed by the phaseindependent multi-modulus algorithm (MMA).

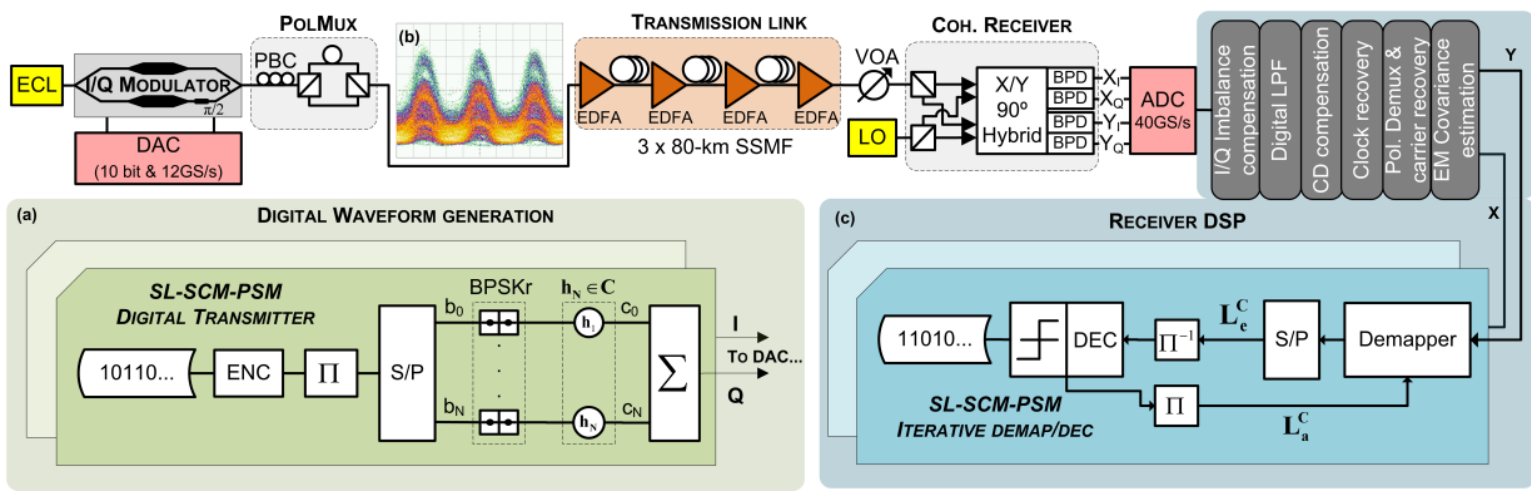

Fig. 3: Experimental setup. Insets: (a) digital transmitter for single-layer SCM-PSM; (b) example of optical eye diagram for 64-PSM; (c) Iterative receiver for single-layer SCM-PSM. 
Carrier recovery is realized with digital phaselocked loop (PLL), where the minimum Euclidean distance metric for symbol decisions is used. For the iterative receiver to work optimally, it is provided with maximum likelihood covariance estimations calculated through the expectation maximization algorithm ${ }^{6}$. For recovering the information bits, a general turbo receiver ${ }^{4,5}$ with an inner demapper and one outer decoder is used. The number of iterations was set to 10 .

\section{Experimental results and discussion}

Fig. 4 shows the bit-error-rate (BER) as a function of signal-to-noise ratio per bit (SNRb) for the measured optical back-to-back after softdecision FEC, for 16-, 32-, and 64- PSM. We thereby show that we can sucesfully demodulate and decode signals employing PSM. For the reference, we also plot the numerically calculated BER and pre-FEC curves.

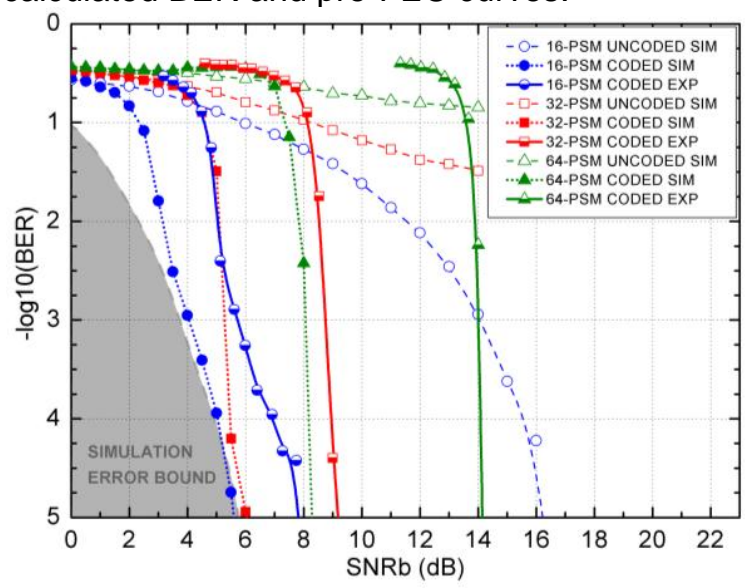

Fig. 1: BER as a function of SNRb, for the measured and simulated optical back-to-back.

Pre-FEC curves prove that coding is mandatory to attain error-free transmission for nonbijective constellations like 32- and 64-PSM. For the proof-of-principle, in this paper, we use $50 \%$ overhead FEC code to rapidly achieve ambiguity resolution yet adding strong coding gain. This permits to analyze the system at very low SNRb while keeping the simplicity of using a single coding block.

The gray area represents the system's error boundary. At such low SNRb, the demapper is unable to provide the decoder with correct enough LLRs, blocking the system and making impossible to reach error-free transmission independently of the number of iterations. This occurs below $\sim 6 \mathrm{dBm}$ SNRb. Despite post-FEC error-free transmission being achieved for all the cases, simulated 16- and 32-PSM cases start to experience error rate limitation at $3.5 \mathrm{dBm}$ and $5.5 \mathrm{dBm}$ respectively. Since implementation penalties $(1.7 \mathrm{~dB}, 3.3 \mathrm{~dB}$ and $6 \mathrm{~dB}$ for 16-, 32and $64-\mathrm{PSM}$ at $10^{-3} \mathrm{BER}$ ) require higher SNRb to obtain similar BER performance, such a limitation is not visible anymore for 32-PSM, whose turbo cliff starts now at $\sim 8 \mathrm{dBm}$. In the 16 -PSM case, the tendency of the boundary is strictly followed given that the penalty is not big enough to exceed the needed SNRb. Note that the implementation penalty also affects the error boundary.

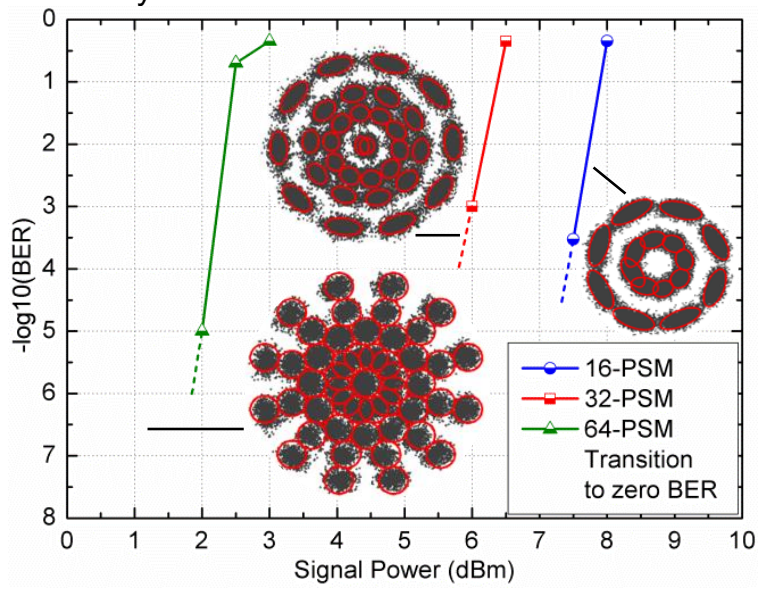

Fig. 2: BER vs. Opt. launched power. 240 km SSMF

Fig. 5 presents the BER as a function of input signal power to each fiber span for a16-, 32- and 64-PSM. Recovered constellations with EMbased covariances for the distorted clusters are also shown. Post-FEC error-free transmission is attained below 2, 6 and $8 \mathrm{dBm}$ for 64-, 32- and 16-PSM respectively.

The results show that joint optimization of the code and modulation level is required. In order to benefit from the shaping gain peak, the turbo cliff must be located in the correct SNR range (see Fig. 2). On the other hand, too strong codes move the cliff to very low SNRs where the system is unable to obtain zero BER (see Fig. 4)

\section{Conclusions}

The first experimental demonstration and transmission of SCM-PSM has been reported. Successful signal demodulation and decoding has been achieved after transmission over 240 $\mathrm{km}$ SSMF. Our results prove the feasibility of using superposition-based modulation schemes in coherent optical communications. Further research is required to find optimum codes taking advantage of the shaping gain while minimizing the overhead. Comparisons to other modulation formats are needed.

\section{References}

[1] P.J.Winzer. J.Lightw.Technol. 30, 24 (2012)

[2] G.Caire, IEEE Trans.Inf.Theory 44, 3 (1998)

[3]I.B.Djordjevic, J.Lightw.Technol 30, 24 (2012)

[4] T.H.Lotz et al, J.Lightw.Technol. 31, 4 (2013)

[5] T.Wo et al., Proc. ITG on SCC (2010)

[6] D. Zibar, Opt.Express 20, 26 (2012). 